\title{
Thermophilic PHB depolymerase of Stenotrophomonas sp., an isolate from the plastic contaminated site is best purified on Octyl-Sepharose CL-4B
}

R Z Sayyed ${ }^{1 *}$, S J Wani ${ }^{1 \&}$, S S Shaikh ${ }^{1 \&}$, Helal F.Al-Harthi", Asad Syed ${ }^{2 \#, ~ H e s h a m ~ A l i ~ E l-~}$ Enshasy\#3,4

${ }^{1}$ Department of Microbiology, PSGVP Mandal's, Arts, Science, and Commerce College, SHAHADA, Maharashtra 425409 India

${ }^{2}$ Department of Botany and Microbiology, College of Science, King Saud University, Riyadh, 11451, Saudi Arabia.

${ }^{3}$ School of Chemical and Energy Engineering, Faculty of Engineering, Universiti Teknologi Malaysia (UTM), Skudai, Johor Bahru, Malaysia,

${ }^{4}$ City of Scientific Research and Technology Applications, New Burg Al Arab, Alexandria, Egypt

\section{*Corresponding author}

R.Z. Sayyed

E-mail: sayyedrz@gmail.com

${ }^{\&}$ These authors contributed equally to this work

\#These authors contributed equally to this work 


\section{Abstract}

There are numerous reports on PHB depolymerases produced by a wide variety of microorganisms isolated from various habitats, however, reports on PHB depolymerase isolated from plastic contaminated sites are scares. Thermophilic PHB polymerase produced by isolates obtained from plastic contaminated sites is expected to have better relevance for its application in plastic/ bioplastic degradation. Although PHB has attracted commercial significance, the inefficient production and recovery methods, inefficient purification of PHB depolymerase and lack of ample knowledge on PHB degradation by PHB depolymerase have hampered its large scale commercialization. Therefore, to ensure the biodegradability of biopolymers, it becomes imperative to study the purification of the biodegrading enzyme system. We report the production, purification, and characterization of extracellular PHB depolymerase from Stenotrophomonas sp. RZS 7 isolated from a plastic contaminated site. The isolate produced extracellular poly- $\beta$-hydroxybutyrate (PHB) depolymerase in the mineral salt medium at $30 \circ \mathrm{C}$ during 4 days of incubation under shake flask condition. Purification of the enzyme was carried out by three different methods using PHB as a substrate. Purification of PHB depolymerase by ammonium salt precipitation, column chromatography, and solvent purification method was successfully carried out. Among the purification method tested, the enzyme was best purified by column chromatography on Octyl-Sepharose CL-4B column with maximum $(0.7993 \mathrm{U} / \mathrm{mg} / \mathrm{ml})$ purification yield. The molecular weight of purified PHB depolymerase (40 kDa) closely resembled with PHB depolymerase of Aureobacterium saperdae.

Keywords: Biodegradable polymer, PHB depolymerase, Thermophilic, Stenotrophomonas sp. 


\section{Introduction}

Poly- $\beta$-hydroxy alkanoates (PHAs) or Poly- $\beta$-hydroxybutyrate (PHB) are accumulated as a source of food and energy by a wide variety of bacteria growing under nitrogen-deficient but conditions and is mobilized during nutrient stress under the influence of PHB depolymerase (16). PHA/PHB is considered as the best eco-friendly and renewable alternative to the synthetics petrochemical plastics because of its similar properties to synthetic plastic (7-8) besides being thermoplastic and biodegradable in nature. Because of such useful properties, it has attracted commercial interest for use as the best alternative to the hazardous synthetic petrochemical polymers and hence it has been successfully commercialized. During last decades much research has been devoted towards distribution and occurrence of PHB degraders and studies on different PHB depolymerases. Jendrossek and Handrick (9) reported that PHB depolymerases are responsible for extracellular PHB degradation. Extracellular PHB depolymerases of Aspergillus fumigatus Pdf1 (10), A. Saperdae (11), Thermus thermophiles HB8 (12), Streptomyces bangladeshensis 77T-4 (13), Penicillium simplicissimum LAR13 (14), Acidovorax sp. TP4 (15), Emericellopsis minima W2 (16) has been isolated and purified.

However, the organism isolated from the plastic contaminated site and having the ability to degrade PHB may be a potential source of dynamic PHB depolymerase. However, inefficient production and recovery process of $\mathrm{PHB}$, inefficient purification of PHB depolymerase and lack of ample knowledge on PHB degradation by PHB depolymerase have hampered the large scale commercialization of PHB. Therefore, to ensure the biodegradability of biopolymers, it becomes imperative to study the purification of biodegrading enzyme system (16). 
The present paper reports the production, purification, and characterization of extracellular PHB depolymerase of Stenotrophomonas sp. RZS 7 isolated from a plastic contaminated site.

\section{Material and Methods}

\section{PHB}

All the experiment was carried out using PHB powder. PHB was obtained from Sigma-Aldrich, Germany.

\section{Source of bacterium}

Stenotrophomonas sp. RZS 7 isolated from plastic contaminated site was previously identified (17) and used in the present study as a source of PHB depolymerase.

\section{Production of PHB depolymerase}

Production of PHB depolymerase was carried out at shake flask level by growing Stenotrophomonas sp. RZS 7 in minimal medium (MM) containing PHB, 0.15\%; $\mathrm{K}_{2} \mathrm{HPO}_{4}, 0.7$ g; $\mathrm{KH}_{2} \mathrm{PO}_{4}, 0.7 \mathrm{~g} ; \mathrm{MgSO}_{4}, 0.7 \mathrm{~g} ; \mathrm{NH}_{4} \mathrm{Cl}, 1.0 \mathrm{~g} ; \mathrm{NaNO}_{3}, 1.0 \mathrm{~g} ; \mathrm{NaCl}, 5 \mathrm{mg} ; \mathrm{FeSO}_{4}, 2 \mathrm{mg}, \mathrm{ZnSO}_{4}$, $7 \mathrm{mg}$ in $1 \mathrm{~L}$ of distilled water (14) at $120 \mathrm{rpm}$ for 4 days at $30^{\circ} \mathrm{C}$.

\section{PHB depolymerase assay}

Following 4 day's incubation at $30^{\circ} \mathrm{C}$ and $120 \mathrm{rpm}, \mathrm{MM}$ broth was centrifuged a $10,000 \mathrm{rpm}$ for 15 min and the supernatant was assayed for PHB depolymerase (12). For this purpose PHB granule (substrate for PHB depolymerase) were sonicated (20 kHz for $15 \mathrm{~min}$ ) and suspended in $50 \mathrm{mM}$ Tris- $\mathrm{HCl}$ buffer ( $\mathrm{pH} 7.0$ ) and $150 \mu \mathrm{g} / \mathrm{ml}$ of this suspension and $2 \mathrm{mM} \mathrm{CaCl}_{2}$ was added in $50 \mathrm{mM}$ Tris-HCl buffer ( $\mathrm{pH}$ 7.0) followed by the addition of culture supernatant (0.5 ml). Enzyme activity was spectrophotometrically measured at $650 \mathrm{~nm}$ as a decrease in the PHB 
turbidity. One unit of PHB depolymerase was defined as the quantity of enzyme required to decrease the absorbance by $0.1 / \mathrm{min}$.

\section{Purification of PHB depolymerase}

Having confirmed the presence of PHB depolymerase in cell-free supernatant of MM broth, the broth was subjected for purification of the enzyme by three approaches as follows

\section{Ammonium salt precipitation}

The crude enzyme in the culture supernatant was precipitated by adding solid ammonium sulfate with continuous stirring at $4^{\circ} \mathrm{C}$ for $1 \mathrm{~h}$. The precipitate was dissolved in the Tris- $\mathrm{HCl}$ buffer $(\mathrm{pH}$ 7) supernatant and dissolved precipitate was transferred in a separate dialysis bag and allowed for overnight dialysis in chilled phosphate buffer (13). The protein concentration of dialyzed supernatant, as well as dialyzed precipitate, was measured by the Lowry method (18). PHB depolymerase activity of dialyzed supernatant and dialyzed precipitate was measured as described earlier.

\section{Solvent purification method}

The culture supernatant of the isolate was centrifuged at 10,000 rpm for $20 \mathrm{~min}$. The residue obtained was dissolved in pre-chilled 1:1 acetone ethanol mixture, shaken well and kept in a water bath at $50^{\circ} \mathrm{C}$ until all solvent is evaporated. The pellet obtained after evaporation was dissolved in Tris-HCl buffer ( $\mathrm{pH}$ 7). PHB depolymerase activity of pellet, as well as supernatant, was carried out as described.

\section{Column chromatography}

The cell-free supernatant of the isolate was applied onto an Octyl-Sepharose CL-4B column preequilibrated with $50 \mathrm{mM}$ glycine $\mathrm{NaOH}$ buffer ( $\mathrm{pH} 9.0$ ) and eluted with a gradient of 0 to $50 \%$ 
ethanol (12), the fractions were collected and PHB depolymerase activity of each fraction was determined as described.

\section{Molecular weight determination of purified PHB depolymerase}

To measure the molecular weight of purified PHB depolymerase, SDS PAGE (Sodium Dodecyl Sulfate Polyacrylamide Gel Electrophoresis) was performed with standard molecular weight markers such as phosphorylase b $(82.2 \mathrm{kDa})$, bovine serum albumin $(64.2 \mathrm{kDa})$, egg albumin (48.8 kDa), carbonic anhydrase $(37.1 \mathrm{kDa})$, trypsin inhibitor $(25.9 \mathrm{kDa})$, lysozyme (19.4 kDa), lysozyme $(14.8 \mathrm{kDa})$ and lysozyme $(6.0 \mathrm{kDa})$. The protein concentration of purified band was measured by the Lowry method with bovine serum albumin (BSA) as a standard (14).

\section{Statistical analysis}

All the experiments were performed in triplicate and the mean of three replicates was considered. Each mean value was subjected to Student's $t$-test and values of $P \leq 0.05$ were taken as statistically significant (19).

\section{Results and Discussion}

\section{Production of PHB depolymerase}

After 4 days' incubation at $30^{\circ} \mathrm{C}$ at $120 \mathrm{rpm}$ in MM, Stenotrophomonas sp. RZS 7 yielded 0.721 $\mathrm{U} / \mathrm{ml}$ PHB depolymerase.

\section{Purification of PHB depolymerase}

\section{Ammonium salt precipitation}

With increasing concentrations of ammonium sulfate in the cell-free culture supernatant of Stenotrophomonas sp. RZS 7, increased precipitation of protein was observed, maximum proteins were precipitated with $70 \%$ ammonium sulfate concentration (Table 1). The protein 
concentration and PHB depolymerase specific activity and enzyme activity of dialyzed precipitate of Stenotrophomonas sp. RZS 7 were $0.219 \mathrm{mg} / \mathrm{ml}, 0.7031 \mathrm{U} / \mathrm{mg} / \mathrm{ml}$ and $0.154 \mathrm{U} / \mathrm{ml}$ respectively. Zhou et al. (20) have reported precipitation of PHB depolymerase of Escherichia coli, and Penicillium sp. DS9701-D2 by using 70 and 75\% of ammonium sulfate Shivkumar et al. (21) have reported efficient precipitation of PHB depolymerase of Penicillium citrinum S2 with $80 \%$ of ammonium sulfate.

\section{Solvent purification method}

Solvent purification of PHB depolymerase of Stenotrophomonas sp. RZS 7, retained only 51.14 $\%$ and $38.81 \%$ enzyme activity in pellet and supernatant (Table 2).

The protein concentration and PHB depolymerase specific activity and enzyme activity in pellet and supernatant were $0.219 \mathrm{mg} / \mathrm{ml}, 0.5114 \mathrm{U} / \mathrm{mg} / \mathrm{ml}$ and $0.3881 \mathrm{U} / \mathrm{ml}$ respectively. This significant loss of enzyme activity may be due to precipitation resulting in denaturation of proteins by solvent system. Thus the solvent purification method proved insignificant vis-à-vis other purification methods.

\section{Column chromatography}

The dialyzed precipitate of Stenotrophomonas sp. RZS 7 obtained after ammonium salt precipitation when applied onto an Octyl-Sepharose CL-4B column pre-equilibrated with $50 \mathrm{mM}$ glycine $\mathrm{NaOH}$ buffer ( $\mathrm{pH}$ 9.0) and eluted with ethanol, yielded 5 fractions. Among all the fractions analyzed for PHB depolymerase enzyme activity, $3^{\text {rd }}$ fractions showed maximum enzyme activity (Table 1). PHBV depolymerase of Bacillus sp. AF3 and Streptoverticillium kashmirense AF1 have also been purified on Sephadex G-75 (22). Kim et al. (23) have also purified PHB depolymerase of Emericellopsis minima W2 and Streptomyces sp. KJ-72 on Sephadex G-100 and Sephadex G-150 respectively. Papaneophytou et al. (12) and Hsu et al. 
(13) have isolated and purified extracellular PHB depolymerases of Thermus thermophiles HB8 and Streptomyces bangladeshensis 77T-4 respectively using column chromatography.

Among all three purification methods, purification on Octyl sepharose CL-4B column gave good purification yield (Table 1), more enzyme activity and more specific activity. The protein concentration and PHB depolymerase specific activity and enzyme activity were $0.247 \mathrm{mg} / \mathrm{ml}$, $0.7993 \mathrm{U} / \mathrm{mg} / \mathrm{ml}$ and $0.309 \mathrm{U} / \mathrm{ml}$ respectively.

\section{Molecular weight determination of purified PHB depolymerase}

The protein fraction of Stenotrophomonas sp. RZS 7 obtained from column chromatography having maximum PHB depolymerase enzyme activity showed single protein band in SDS PAGE having a molecular mass of approximately $40 \mathrm{kDa}$ lying between molecular weight markers of 37.1 and 48.8 (Fig 1). Sadocco et al. (11) have also reported the molecular mass of PHB depolymerase from Aureobacterium saperdae ranges from $42.7 \mathrm{kDa}$ analyzed by SDS PAGE.

\section{Conclusion}

The present attempt provided PHB degrading Stenotrophomonas sp. RZS 7 which utilized PHB as a sole source of carbon under the influence of extracellular PHB depolymerase. The enzyme acted as a key enzyme responsible for biodegradation of PHB. Purification of PHB depolymerase by solvent purification resulted in protein precipitation and denaturation of the enzyme. Column chromatography by using octyl-Sepharose CL-4B came out as efficient and best purification method as it purification yielded maximum protein, maximum enzyme activity, and maximum specific activity. The molecular weight of purified PHB depolymerase of Stenotrophomonas sp. RZS $7(40 \mathrm{kDa})$ matched with a molecular weight of Aureobacterium saperdae.

\section{Competing interests}


All authors declare no conflict of interest.

\section{Author Contributions}

Conceptualization and drafting: R. Z. Sayyed

Investigation: S. J. Wani and S S Shaikh

Data curation: Abdullah A. Alyousef, Abdulaziz Alqasim

Funding acquisition: Abdullah A. Alyousef, Abdulaziz Alqasim, Hesham Ali El-Enshasy

Data Availability Statement: All relevant data are within the paper.

\section{Funding.}

This research is supported by grant No. RG-1440-053 by the Deanship of Scientific Research at King Saud University, Saudia Arabia and the support of MOE and UTM-RMC (Malaysia) through HICOE grant No. R.J130000.7846.4J262. 


\section{References}

1. Sayyed RZ, Gangurde NS, Chincholkar SB, Hypochlorite digestion method for efficient recovery of PHB from A. feacalis, Indian J Microbiol, 2009; 49: 230-232.

2. Gangurde NS, Patil YP, Jain R, Sayyed RZ, Poly- $\beta$-hydroxybutyrate biodegradation by mixed culture population vis-à-vis single culture population under varying environmental conditions: a new approach, Indian J Exp Biol, 2017; 55: 311-320.

3. Wani SJ, Shaikh SS, Tabassum B, Thakur A, Gulati A, Stenotrophomonas sp. RZS7, a novel PHB degrader isolated from plastic contaminated soil in Shahada, Maharashtra, Western India, 3 Biotech, 2016; 6: 179.

4. Wani SJ, Sayyed RZ, Production, efficient recovery and partial characterization of biodegradable polymer produced by soil Streptomyces, Indian J Biotechnol, 2016; 15: 127129.

5. Gangurde NS, Sayyed RZ, Shashi K, Gulati A, Development of eco-friendly bioplastic like PHB from distillery effluent microorganisms, Env Sci Poll Res, 2013, 20: 488-497.

6. Soam A, Singh A, Singh R, and Shahi S, Optimization of culture conditions for biopolymer producing Bacillus mycoides (wss2) bacteria from sewage. Current discovery 2012, 1: 2732.

7. Kumar MS, Mudliar SN, Reddy KMK and Chakraborti T, Production of Biodegradable Plastic from Activated Sludge Generated from the Food Processing Industrial Wastewater Treatment Plant, Bioresource Technology, 2004 95: 327-330.

8. Sudesh K, Abe H, and Doi Y, Synthesis and properties of polyhydroxyalkanoates: biological polyesters. Progress in Polymer Science, 2000, 25: 1503-1555. 
9. Jendrossek D and Handrick R, Microbial degradation of polyhydroxyalkanoates. Ann. Rev. Microbiol 2002, 56: 403-432.

10. Iyer S, Shah R, Sharma A, Jendrossek D and Desai A, Purification of Aspergillus fumigatus (Pdf1) poly( $\beta$-hydroxybutyrate) (PHB) depolymerase using a new, single-step substrate affinity chromatography method: characterization of the PHB depolymerase exhibiting novel self-aggregation behavior. J Polym Environ 2000, 8: 197-203.

11. Sadocco PS, Nocerino E, Dubini-Paglia A and Seres EG, Characterization of a poly(3hydroxybutyrate) depolymerase from Aureobacterium saperdae : Active site and kinetics of hydrolysis studies. J. Environ. Polym. Degrad 1997, 5: 57-65.

12. Papaneophytou CP, Pantazaki AA and Kyriakidis DA, An extracellular polyhydroxybutyrate depolymerase in thermus thermophilus hb8. Appl Microbiol Biotechnol, 2009, 83: 659-668.

13. Hsu KJ, Tseng M, Don TM and Yang MK, Biodegradation of Poly( $\beta$-hydroxybutyrate) by a novel isolate of Streptomyces bangladeshensis 77T-4. Botanical studies, 2012, 53:307313.

14. Han JS and Kim MN, Purification and characterization of extracellular Poly (3hydroxybutyrate) Depolymerase from Penicillium simplicissimum LAR13. The Journal of Microbiology 2002, 40: 20-25.

15. Kobayashi T, Sugiyama A, Kawase Y, Saito T, Mergaert J and Swings J (1999) Biochemical and genetic characterization of an extracellular Poly(3-Hydroxybutyrate) depolymerase from Acidovorax Sp. Strain TP4. J Env Pol Deg 1999, 7: 9-18. 
16. Kim HJ, Nam JS, Bae KS and Rhee YH, Characterization of an extracellular medium-chain length poly (3-hydroxyalkanoate) depolymerase from Streptomyces sp. KJ-72, Antony van Leeuwenhoek 2003, 83: 183-189.

17. Wani SJ, Shaikh SS and Sayyed RZ, Medium optimization for PHB depolymerase production by Stenotrophomonas maltophilia using Plackett Burman design \& response surface methodology. Intntl J of Scientific \& Engg Res, 2015, 6: 818-829.

18. Lowry OH, Rosebrough NJ, Farr AL and Randall RJ, Protein measurement with the Folin phenol reagent. J Biol Chem, 1951, 193: 265-275.

19. Zhou H, Wang Z, Chen S, Liu D and Xia H, Purification and characterization of extracellular poly( $\beta$-hydroxybutyrate) depolymerase from Penicillium sp. DS9701-D2. Polymer-Plastics Technol Engg, 2008, 48: 58-63.

20. Shivakumar S, Jagadish SJ, Zatakia H, and Dutta J, Purification, characterization and kinetic studies of a novel poly $(\beta)$ hydroxybutyrate (PHB) depolymerase PhaZ Pen from Penicillium citrinum S2. Appl Biochem and Biotechnol, 2011, 164: 1225-1236

21. Shah AA, Hasan F, Hameed A, Ahmed S (2007) Isolation and characterization of poly (3hydroxybutyrate-co-3-hydroxy valerate) degrading actinomycetes and purification of PHBV depolymerase from newly isolated Streptoverticillium kashmirense AF1. Annals of Microbiol 2007, 57: 583-588

22. Kim DY, Yun JH, Kim HW, Bae KS, and Rhee YH, Purification and characterization of poly (3-hydroxybutyrate) depolymerase from a fungal isolate, Emericellopsis minima W2. J Microbiol 2002, 40: 129-133.

23. Parker RE, Continuous distribution: tests of significance. In: Parker RE (ed) Introductory statistics for biology, 2nd Edn; 1979; Cambridge University Press, London, pp 18-42. 
Table 1 - Purification of PHB depolymerase of Stenotrophomonas sp. RZS7 on Octyl sepharose column

\begin{tabular}{|c|c|c|c|c|}
\hline Fraction & $\begin{array}{c}\text { Total activity } \\
(\mathbf{U} / \mathbf{m l})\end{array}$ & $\begin{array}{c}\text { Total protein } \\
(\mathbf{m g} / \mathbf{m l})\end{array}$ & $\begin{array}{c}\text { Specific activity } \\
(\mathbf{U} / \mathbf{m g} / \mathbf{m l})\end{array}$ & \% activity \\
\hline 1 & $0.027(0.027)$ & $0.031(0.010)$ & $0.0782(0.032)$ & $11.13(0.012)$ \\
\hline 2 & $0.101(0.011)$ & $0.153(0.037)$ & $0.3781(0.041)$ & $48.31(0.039)$ \\
\hline 3 & $0.247(0.015)$ & $0.309(0.074)$ & $0.7993(0.090)$ & $79.93(0.092)$ \\
\hline 4 & $0.017(0.001)$ & $0.015(0.009)$ & $0.0106(0.011)$ & $04.01(0.012)$ \\
\hline 5 & $0.003(0.001)$ & $0.006(0.005)$ & $0.0011(0.008)$ & $00.10(0.002)$ \\
\hline
\end{tabular}

Values were taken to be statistically significant at $\mathrm{P} \leq 0.05$

Values are the mean of three replicates 
Table 2 - Purification profile of PHB depolymerase by various methods

\begin{tabular}{|c|l|c|}
\hline \multicolumn{1}{|c|}{ Isolate } & \multicolumn{1}{|c|}{ Purification method } & Enzyme activity (U/mg/ml) \\
\hline \multirow{3}{*}{ Stenotrophomonas sp. RZS 7 } & Salt precipitation & $0.7031(0.090)$ \\
\cline { 2 - 3 } & Solvent purification & $0.1120(0.022)$ \\
\cline { 2 - 3 } & Column chromatography & $0.7993(0.099)$ \\
\hline
\end{tabular}

Values were taken to be statistically significant at $\mathrm{P} \leq 0.05$

Values are the mean of three replicates 